Check for updates

Cite this: RSC Adv., 2017, 7, 37086

Received 17th February 2017

Accepted 12th June 2017

DOI: 10.1039/c7ra02020a

rsc.li/rsc-advances

\section{The effect of a human acellular amniotic membrane loaded with mechanical stretch-stimulated bone marrow mesenchymal stem cells for the treatment of pelvic floor dysfunction}

\author{
Bing Zhao, $\dagger^{\mathrm{a}}$ Linlin Liang, $\dagger^{\mathrm{b}}$ Junmin Wang, (DD ac Chenchen Ren, ${ }^{a}$ Mengcai Hu, ${ }^{a}$ \\ Huiyan $\mathrm{Wu}{ }^{a}$ Lulu Chen, ${ }^{a}$ Xiaojun Liu, ${ }^{d}$ Feng $\mathrm{Xu}{ }^{a}$ Xueqin Zheng, ${ }^{a}$ Juan $\mathrm{Chen}^{a}$ \\ and Shihong Cui ${ }^{\star a}$
}

Pelvic floor dysfunction (PFD) has a severe impact on the quality of life of middle-aged and elderly women and is closely related to the damage of pelvic support tissues, especially ligaments. The incidence of PFD is high in elderly populations. Conventional treatments are associated with high complication and relapse rates. Bone marrow mesenchymal stem cells (BMSCs) are bone marrow-derived pluripotent stem cells that can differentiate into many types of cells. We have found that when cultured with mechanical stretch-stimulated pelvic ligament fibroblasts, rat BMSCs were induced to differentiate into pelvic ligament fibroblasts. Human acellular amniotic membranes (HAAMs) demonstrate a good biocompatibility and can promote BMSC proliferation and BMSC differentiation into ligament fibroblasts. In this study, BMSCs cultured with mechanical stretch-stimulated pelvic ligament fibroblasts were inoculated into and cultured onto HAAMs, which were then implanted into rats with PFD to improve PFD symptoms. These results indicated their potential use as a cell-based therapy for PFD.

\section{Introduction}

Pelvic floor dysfunction (PFD) refers mainly to pelvic organ prolapse (POP) and stress urinary incontinence (SUI). PFD, which is a common condition in middle-aged and elderly women, has a severe impact on the health and quality of life of those women and has an incidence of approximately $40 \%{ }^{1,2}$ Although not life-threatening, PFD-related urinary incontinence, difficulty urinating, and abdominal dragging sensations affect the physical health and well-being of affected women, and have a severe impact on health and quality of life. PFD is a complex, multifactorial condition that is closely related to the structural and functional integrity of pelvic support tissues (ligaments, muscles, fascia), especially the pelvic uterosacral ligament-uterine cardinal ligament complex. ${ }^{2,3}$ Currently, PFD treatments include non-surgical treatment for mild cases and surgical treatment for severe cases. During surgery, however,

${ }^{a}$ Third Affiliated Hospital of Zhengzhou University, Zhengzhou, Henan Province 450052, PR China. E-mail: shihongcui@126.com; Tel: +8613613809826

${ }^{b}$ The Center for Reproduction, Henan Province People's Hospital, Zhengzhou, Henan Province 450052, PR China

${ }^{c}$ Laboratory Animal Center, Zhengzhou University, Zhengzhou, Henan Province 450052, PR China

${ }^{d}$ Henan Medical Equipment Inspection Institute, Zhengzhou, Henan Province 450052, PR China

$\dagger$ Contributed equally to this work. normal tissues are often used to repair tissue defects, which results in a poor outcome, high complication and relapse rates, and problems such as a high risk of infection, high cost, and high incidence of erosion, leakage, and rejection. ${ }^{4-6}$

With the rise in popularity of tissue engineering and its successful application in different clinical areas, new treatments for PFD are emerging. Tissue engineering is very promising for PFD caused by tissue and organ dysfunction and defects. As seed cells for tissue engineering, bone marrow mesenchymal stem cells (BMSCs) are derived from the mesoderm, and thus, they have potent self-replication and multipotent differentiation potentials. BMSCs are involved in the induction and regulation of the development of bone marrow hematopoietic stem cells and stromal cells and promote the orderly arrangement of extracellular matrix molecules. In cases of tissue and cell damage, both endogenous and exogenous BMSCs migrate to the lesion, where they play a role in repair. ${ }^{7}$ Our pilot study first showed that when cultured with mechanical stretch-stimulated pelvic ligament fibroblasts, rat BMSCs were induced to differentiate into pelvic ligament fibroblasts, ${ }^{8}$ which could repair PFD caused by damage to support tissues, such as pelvic floor ligaments, fascia, and muscles. Human acellular amniotic membrane (HAAM) has been used as a biological material in basic research and clinical practice for many years. HAAM is an ideal bio-scaffold that promotes BMSC adhesion, growth, and proliferation, and thus, it contributes to tissue repair. Some researchers have used stem 
cell-loaded acellular amniotic membrane to repair skin tissue defects., ${ }^{910}$ This indicates that BMSC-loaded HAAM may be a viable and good option for the treatment of PFD.

In this study, we cultured rat BMSCs with mechanical stretch-stimulated pelvic ligament fibroblasts to induce BMSC differentiation into pelvic ligament fibroblasts within a specific in vitro mechanical microenvironment. Once BMSC differentiation was confirmed based on the levels of collagen, elastin, lysyl oxidase (LOX), and fibulin-5, HAAM was inoculated with well-differentiated BMSCs, which were then cultured on HAAM. Finally, BMSC-loaded HAAM was implanted into rats with PFD in order to observe its effect on PFD symptoms.

\section{Materials and methods}

\section{Isolation, culture, and identification of BMSCs}

The femur and tibia were removed from eight-week-old male rats, and DMEM low-glucose medium was used to flush the bone marrow into a Petri dish. This study was performed in strict accordance with the NIH guidelines for the care and use of laboratory animals (NIH Publication No. 85-23 Rev. 1985) and was approved by the Institutional Animal Care and Use Committee of Zhengzhou University (Zhengzhou, China). Next, complete medium (also known as basal medium containing DMEM lowglucose medium with $10 \%$ fetal bovine serum (FBS) and $1 \%$ penicillin and streptomycin) was added; after the bone marrow was cultured at $37{ }^{\circ} \mathrm{C}$ in an atmosphere of $5 \% \mathrm{CO}_{2}$ and saturated humidity for 3 days, non-adherent cells were removed, and the remaining cells were cultured and passaged three times (P3). Then, flow cytometry was used to detect cell surface markers, and multipotent differentiation potential was confirmed. To induce osteogenic differentiation, BMSCs were seeded into cell culture plates at a density of $1 \times 10^{4}$ cells per $\mathrm{cm}^{2}$, and osteogenic induction solution was added. In the control group, complete medium was added in place of osteogenic induction solution. The cells were cultured for 21 days and then stained with alizarin red. To induce chondrogenic differentiation, BMSCs were prepared as a cell suspension $\left(1 \times 10^{7}\right.$ cells per $\left.\mathrm{mL}\right)$, and then a drop $(10 \mu \mathrm{L})$ was added to each well of 12 -well plates. The cells were maintained in an incubator for 2 hours to allow the cells to adhere, and then chondrogenic induction solution was slowly added. In the control group, complete medium was added in place of chondrogenic induction solution. The cells were cultured for 28 days and then stained with toluidine blue. To induce adipogenic differentiation, BMSCs were seeded into cell culture plates at a density of $1 \times 10^{4}$ cells per $\mathrm{cm}^{2}$. In the induction and differentiation group, adipogenic induction solution was added, and the cells were induced for 2 days. Next, adipogenic induction maintenance solution was added, and the cells were further induced for 1 day. The cells were cultured for 14 days and then stained with oil red $\mathrm{O}$. In the control group, complete medium was added in place of adipogenic solution.

\section{Western blotting}

PMSF-containing RIPA lysis buffer was used to lyse cells on ice for 30 minutes, followed by centrifugation at $4{ }^{\circ} \mathrm{C}$ at
$12000 \mathrm{rpm}$ for 10 minutes to collect the supernatant. The protein samples collected were separated via $10 \%$ SDS gel electrophoresis. After electrophoresis, the proteins were transferred to a PVDF membrane, blocked in 5\% BSA, incubated with the primary antibody at $4{ }^{\circ} \mathrm{C}$ overnight, incubated with an HRP-labeled secondary antibody, and then detected with ECL reagents. The primary antibodies used were as follows:

\section{Real-time fluorescence quantitative polymerase chain reaction (PCR)}

The total RNA of the cells was isolated using TRIzol reagent (Invitrogen) according to the manufacturer's instructions. After the reverse transcription reaction, real-time PCR was performed in an ABI 7900HT system using SYBR Premix (Takara, Dalian, China) according to the manufacturer's instructions. The conditions of the real-time PCR were as follows: denaturation at $95{ }^{\circ} \mathrm{C}$ for $10 \mathrm{~s}, 40$ cycles at $95{ }^{\circ} \mathrm{C}$ for $10 \mathrm{~s}$ and $60^{\circ} \mathrm{C}$ for $30 \mathrm{~s}$. A dissociation stage was added to the end of the amplification procedure. No nonspecific amplification was observed, as determined by the dissociation curve. Glyceraldehyde 3-phosphate dehydrogenase (GAPDH) was used as an internal control. The data were analyzed using the comparison $C_{\mathrm{t}}\left(2^{-\Delta \Delta C_{\mathrm{t}}}\right)$ method and were expressed as the fold change relative to the respective control. Each sample was analyzed in triplicate. The primer sequences used in this study were as follows:

\section{Immunohistochemistry}

BMSCs cultured in a dish were fixed in 4\% PFA in PBS for $20 \mathrm{~min}$ at room temperature. After washing in PBS, the samples were permeabilized with $0.5 \%$ Triton $\mathrm{X}-100$ for $5 \mathrm{~min}$ and blocked with $5 \%$ BSA for $60 \mathrm{~min}$. Incubation with primary anticollagen I and anti-collagen III antibodies (Abcam) was performed overnight at $4{ }^{\circ} \mathrm{C}$. The primary antibodies were detected using HRP-conjugated anti-rabbit or mouse IgG secondary antibodies and DAB.

\section{CCK-8 assay to determine viability of BMSCs}

HAAM was cut into small pieces, which were then placed in the wells of 96-well plates. HAAM was inoculated with BMSCs at a density of $1 \times 10^{4}$ cells per well. Next, $10 \mu \mathrm{L}$ of CCK-8 assay solution was added to each well. After the cells were incubated at $37{ }^{\circ} \mathrm{C}$ for 1 hour, the medium was aspirated, and the $\mathrm{OD}_{450}$ was measured in a microplate reader.

\section{Labeling of BMSCs with CM-DiI}

BMSCs were prepared as a cell suspension in PBS $\left(1.0 \times 10^{7}\right.$ cells per $\mathrm{mL})$. Next, $40 \mu \mathrm{L}$ of CM-DiI $\left(1 \mathrm{mg} \mathrm{mL}^{-1}\right)$ was added per $1 \mathrm{~mL}$ of cell suspension (final concentration of CM-DiI: $40 \mu \mathrm{M}$ ). The cell suspension was placed at $37^{\circ} \mathrm{C}$ for 3 minutes and then in an ice bath for 15 minutes. Labeled BMSCs were used in subsequent experiments and were tracked under a fluorescence microscope. 


\section{Rat PFD model}

Post-partum SD rats were anesthetized via an intraperitoneal injection of isobarbital. Next, the rats were secured in a prone position; after the bladder was emptied with a catheter, a doublelumen Foley catheter was placed into the vagina. The balloon was inflated with sterile sodium chloride solution until the pubic symphysis abutted the edge of the table. The catheter dropped freely, and a force of $0.15 \mathrm{~kg}$ was applied for 4 hours. Next, the catheter was removed, and the rats were returned to their cage. One month later, bilateral oophorectomy was performed with aseptic technique, and the rats were returned to their cage after the surgery. Penicillin was given via intraperitoneal injection, qd, for 5 days. The rats were further housed for 1 month.

\section{Function of PFD rats}

After anesthesia was induced by an intramuscular injection of ketamine, the rats were secured in a supine position for urodynamic testing. The bladder was emptied, and then $0.9 \%$ sodium chloride solution with methylene blue $(50 \%$ of the measured bladder capacity) was injected via a micro-pump. The rats were carefully monitored for blue solution flowing from the urethra during sneezing. The result was considered positive in rats with urine flow and negative otherwise; the positive rate was then calculated.

\section{Statistical analysis}

The data are presented as the mean \pm S.D. ( $n$ is the number of tissue preparations, cells or experimental replicates). A twotailed Student's $t$-test was used to compare groups of data. A value of $P<0.05$ was considered statistically significant.

\section{Results and discussion}

\section{Isolation and identification of BMSCs}

BMSCs are bone marrow-derived pluripotent stem cells with multipotent differentiation potential. Under certain conditions, BMSCs can be induced to differentiate into adipocytes, osteoblasts, and chondrocytes. To investigate how well BMSCs may differentiate into ligament fibroblasts, we first identified
A

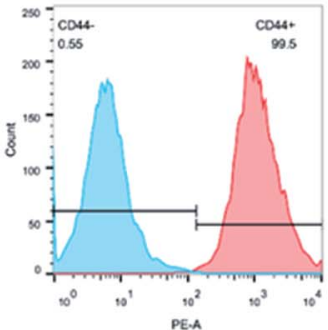

CD $4499.5 \%$

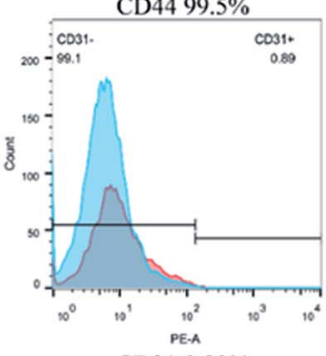

CD31 0.89\%

B
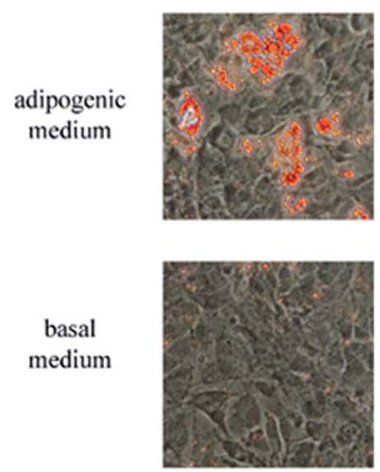

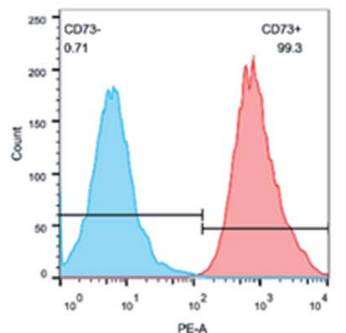

CD73 $99.3 \%$

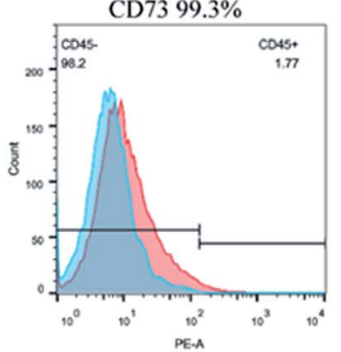

CD $451.77 \%$

C
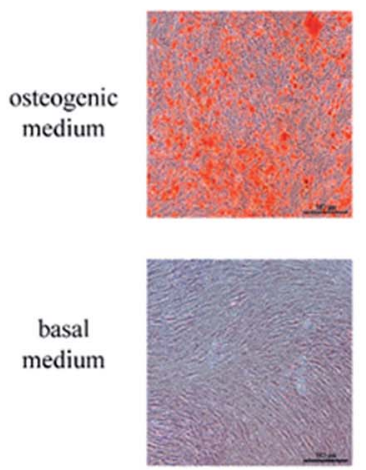

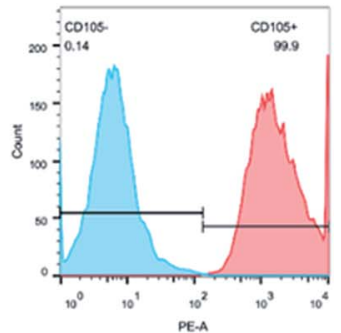

CD105 99.9\%
D

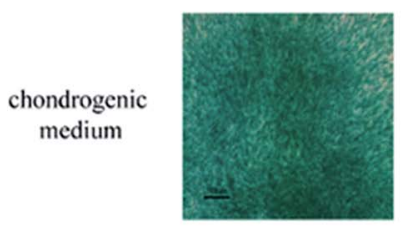

basal medium

Fig. 1 Identification of BMSCs. (A) The expression of BMSC markers CD44, CD73, and CD105; endothelial cell surface marker CD31; and leukocyte surface marker CD45 on BMSCs. (B) BMSCs were cultured with adipogenic medium (or complete medium in the control group) for 14 days and stained with oil red $\mathrm{O}$. (C) BMSCs were cultured with osteogenic medium (or complete medium in the control group) for 21 days and then stained with alizarin red. (D) BMSCs were cultured with chondrogenic medium (or complete medium in the control group) for 28 days and then stained with toluidine blue. 
isolated BMSCs. Flow cytometry showed that the BMSC markers CD44, CD73, and CD105 were highly expressed on isolated BMSCs, with little expression of the endothelial cell marker CD31 and the leukocyte surface marker CD45 (Fig. 1A). Furthermore, we tested the multipotent differentiation potential of isolated BMSCs and showed that isolated BMSCs were capable of differentiating into adipocytes, osteoblasts, and chondrocytes (Fig. 1B-D). These results indicated a high purity level of isolated BMSCs. PFD is a complex, multifactorial, and multi-stage condition. The mechanism of PFD is unknown, but researchers believe that $\mathrm{PFD}$ is closely related to damage of the structural and functional integrity of pelvic support tissues, including multiple layers of muscles, ligaments, and fascia that close the pelvic outlet. ${ }^{11,12}$ With the rapid development of tissue engineering and stem cell therapy and their successful applications in clinical practice, new PFD treatments are emerging. BMSCs are cells with multipotent differentiation potential. They are easy to isolate and culture, have good allogeneic immune responses, and can readily proliferate in vitro. As a result, they have attracted a large amount of attention; in particular, numerous studies have investigated the potential of BMSCs in the field of tissue and organ repair. ${ }^{13-15}$ In this study, we used a cell adhesion method to isolate rat BMSCs.

\section{Co-cultured with mechanical stretch-stimulated pelvic ligament fibroblasts promote rat BMSCs differentiated into pelvic ligament fibroblasts}

Our pilot study showed that mechanical stretch could alter the morphology of pelvic ligament fibroblasts. ${ }^{8}$ This study further showed that when cultured with mechanical stretch-stimulated pelvic ligament fibroblasts, rat BMSCs expressed a high level of elastin, LOX, and fibulin-5 (Fig. 2A-C), while immunohistochemical staining showed that these rat BMSCs expressed a high level of type I collagen (Fig. 2D) and type III collagen (Fig. 2E). These results indicated that when cultured with mechanical stretch-stimulated pelvic ligament fibroblasts, rat BMSCs were induced to differentiate into pelvic ligament
A

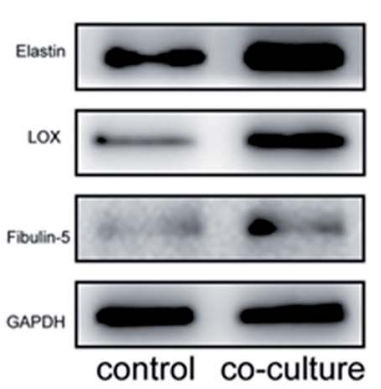

$\mathrm{C}$

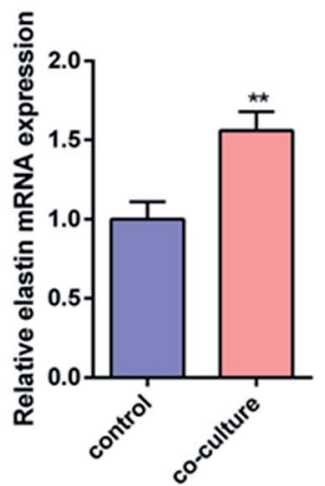

D

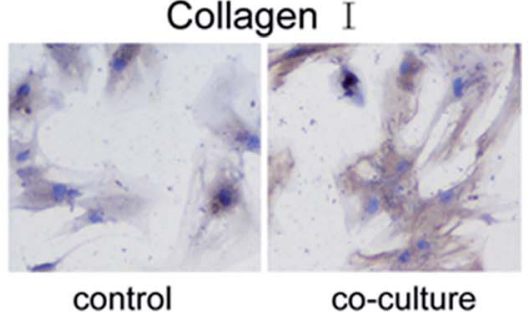

B
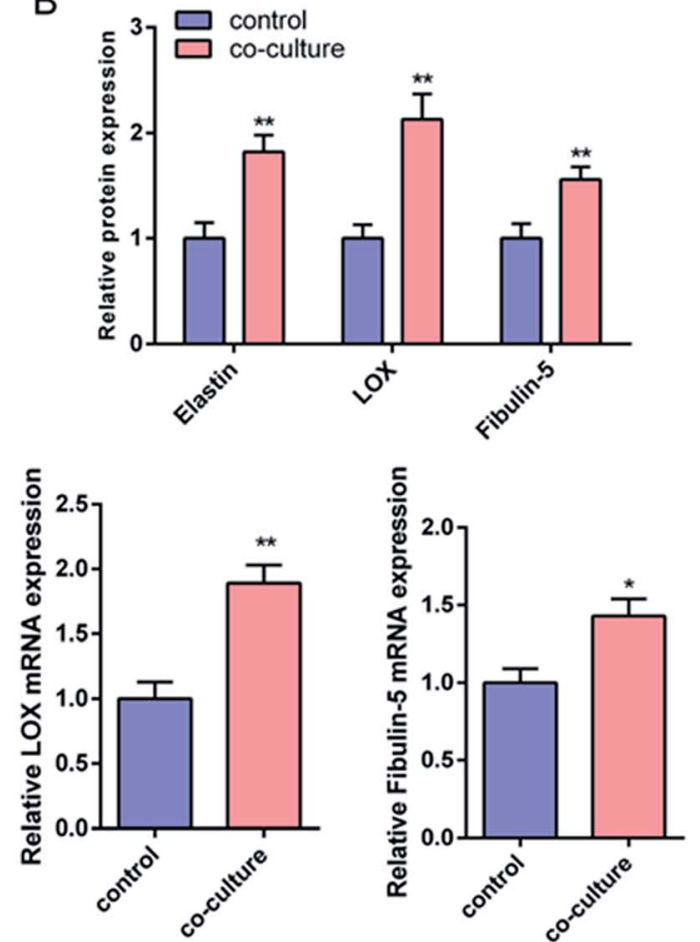

E

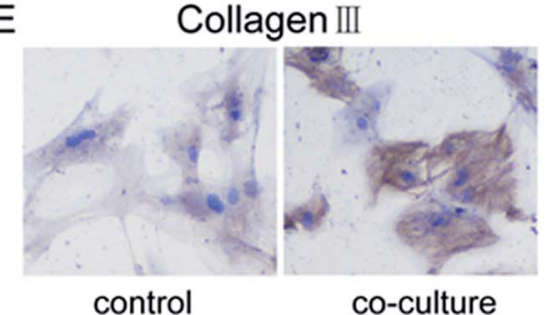

Fig. 2 Cultured with mechanical stretch-stimulated pelvic ligament fibroblasts, rat BMSCs differentiated into pelvic ligament fibroblasts with base medium. (A) Western blotting analysis of elastin, LOX and fibulin-5 expression in BMSCs. (B) Gray-scale analysis of western blotting results. (C) Real-time PCR detection of elastin, LOX and fibulin-5 expression in BMSCs. Immunohistochemistry staining for type I collagen (D) and type III collagen (E) on BMSCs. ${ }^{*} P<0.05, * * P<0.01$. 
fibroblasts. Numerous studies have shown that during repair, BMSCs are found in the local area of ligament damage. Some researchers injected BMSCs from transgenic rats into the area of ligament damage of wild-type rats; 28 days later, live BMSCs were detected in the healed ligament of wild-type rats, and the morphology of these BMSCs was similar to that of the surrounding ligament fibroblasts. These results suggested that the injection of BMSCs in the area of ligament damage could significantly improve the repair ability of the ligament without alterations in the microstructure of the tissue. Thus, BMSCs are considered an effective cellular carrier that can be used to deliver a therapeutic effect to a damaged ligament while improving the speed and quality of repair. ${ }^{\mathbf{1 6}}$ Some researchers have used 5-Aza to induce BMSCs to differentiate into fibroblasts, which provides a source of seed cells for injection of female patients with PFD. Some researchers injected BMSCs into SUI rats; during the study, undifferentiated or differentiated BMSCs were injected into the mucosa, muscle of the bladder inlet, or the posterior urethra of SUI rats. They found that, after injection, the maximum bladder capacity was significantly increased and that the positive rate of the sneeze test and maximum detrusor pressure were significantly decreased compared with before the injection. ${ }^{17}$ This study showed that co-culture of mechanical stretch-induced pelvic ligament fibroblasts with BMSCs promoted the differentiation of BMSCs into ligament fibroblasts, but further research is required to investigate the physiological mechanism.

\section{HAAM promoted the proliferation of BMSCs}

More and more researchers are investigating the use of HAAM as a cell scaffold. In this study, we first inoculated HAAM with BMSCs and then analyzed the effect of HAAM on the proliferation of BMSCs after 1, 3, and 7 days. A CCK-8 assay showed that HAAM promoted the proliferation of BMSCs (Fig. 3A) but had no effect on BMSC apoptosis (Fig. 3B). Laser confocal microscopy showed that the number of BMSCs on HAAM increased over time (Fig. 3C) and that BMSCs were well distributed over HAAM (Fig. 3D). Due to its special structure and components, amniotic membrane induces the growth of certain cells and is thus often used as the vehicle for cell growth and proliferation. In recent years, many researchers have cultured amniotic membrane with cells. ${ }^{\mathbf{1 0 , 1 8 , 1 9}}$ Some studies showed that 8 days after they were seeded on HAAM, BMSCs showed a radial, swirling, or parallel growth pattern. The cell body was hypertrophic with an irregular, long polygonal, flat shape and contained a large number of projections, some of which were woven into a network. The cells were also securely adhered to the surface of the amniotic membrane matrix. Once loaded with an appropriate density of BMSCs, the HAAM-BMSC complex becomes a dermal substitute that is suitable for implantation. ${ }^{20}$ In this study, we seeded HAAM with BMSCs and found that HAAM promoted the proliferation of BMSCs and that BMSCs adhered well to HAAM.
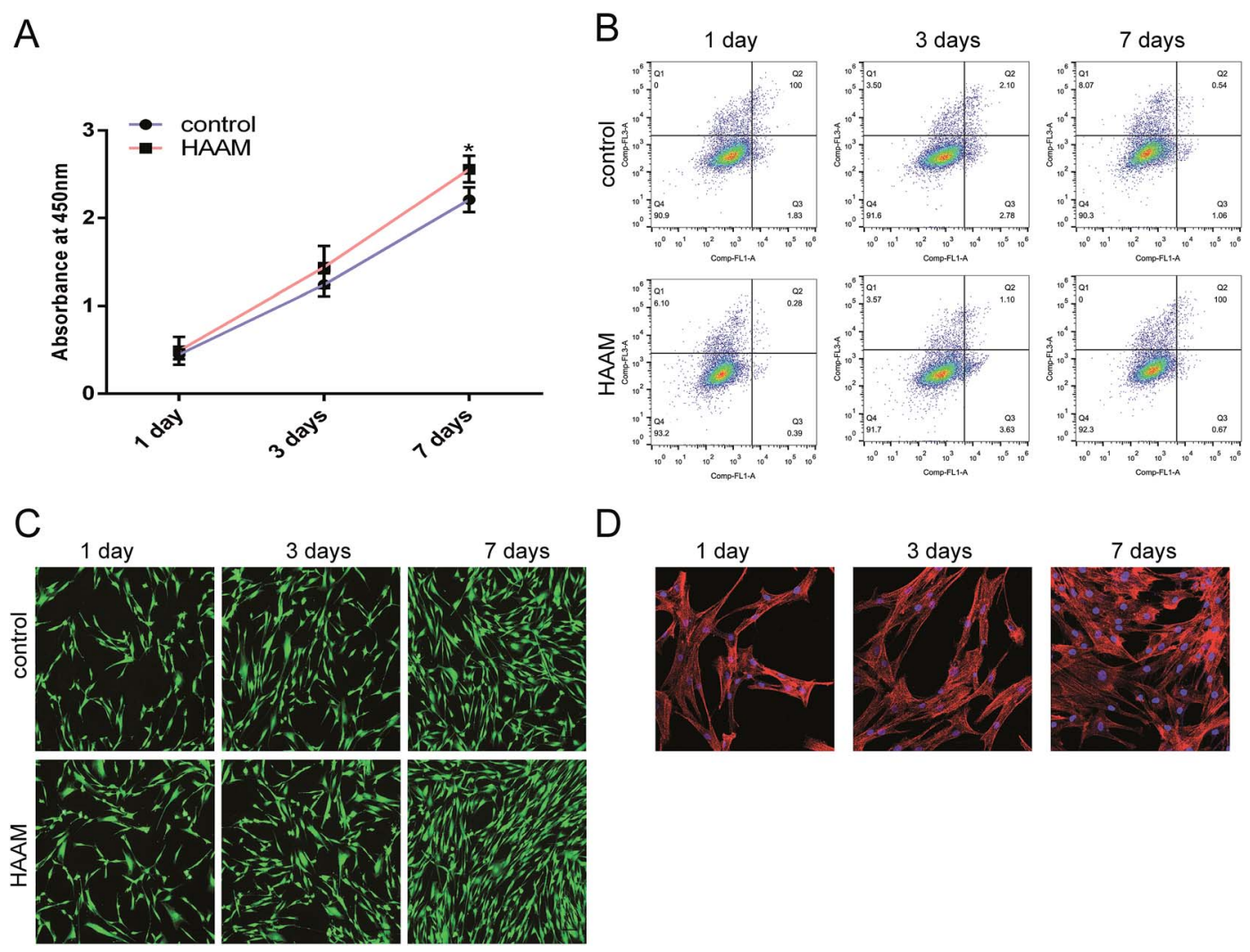

Fig. 3 HAAM promoted the proliferation of BMSCs. (A) CCK-8 assay of the effect of HAAM on BMSC proliferation. (B) Flow cytometry of the effect of HAAM on BMSC apoptosis. (C) Calcein staining of the effect of HAAM on the proliferation of BMSCs. (D) Laser confocal microscopy of the spread of BMSCs on HAAM. Phalloidin (red), blue (Hoechst 3342) $* P<0.05, * * P<0.01$. 
HAAM promoted the differentiation of BMSCs into pelvic ligament fibroblasts

HAAM is highly compatible with BMSCs. To date, no studies have been conducted to investigate the effect of HAAM on the differentiation of BMSCs into ligament fibroblasts. In this study, after HAAM was inoculated with BMSCs and cultured with them for 0 , 3,7 , or 14 days, cell samples were collected and analyzed. Western blotting showed that the expression of elastin, LOX, and fibulin-5 in BMSCs increased over time (Fig. 4A). Real-time PCR revealed that the mRNA levels of elastin, LOX, and fibulin-5 in BMSCs were also increased over time (Fig. $4 \mathrm{~B}$ and C). As in our earlier studies, ${ }^{8}$ the expression of elastin, LOX and fibulin-5 did not change significantly when BMSCs were cultured in vitro for a long time individually, no negative control experiment was done for this present work. These results suggested that culture on HAAM effectively promoted the differentiation of BMSCs into ligament fibroblasts.

HAAM loaded with BMSCs cultured with mechanical stretchstimulated pelvic ligament fibroblasts promoted the production of ligament fibrous tissue in rats

Currently, available PFD treatments are associated with a poor outcome as well as high complication and relapse rates. Therefore, it is important to develop new treatments. With the rise in popularity of tissue engineering and its successful application in different clinical areas, new PFD treatments are emerging. Tissue engineering is very promising for PFD caused by tissue and organ dysfunction and defects. In this study, we cultured BMSCs with mechanical stretch-stimulated pelvic ligament fibroblasts and then seeded BMSCs on HAAM for implantation into PFD rats. Specifically, we isolated and purified BMSCs, cultured BMSCs with mechanical stretchstimulated pelvic ligament fibroblasts, labeled BMSCs with CM-DiI, and then inoculated HAAM with BMSCs. Then, BMSCloaded HAAM was implanted around the urethra of PFD rats. In this study, PFD rats were randomly divided into three groups, each containing 6 rats. In the control group, HAAM was implanted around the urethra; in the 2 experimental groups, HAAM loaded with undifferentiated or differentiated BMSCs was implanted. BMSCs were first labeled with CM-DiI and then passaged in vitro; a fluorescence microscope was used to observe the fluorescence intensity of the dye in BMSCs. The results showed that BMSCs were well labeled with high fluorescence intensity even after several passages (Fig. 5A). HAAM loaded with CM-DiI-labeled BMSCs was implanted into PFD rats. Three weeks later, the HAAM was removed, and fluorescence microscopy revealed a large number of live BMSCs on the HAMM (Fig. 5B). Moreover, HAAM loaded with BMSCs cultured with mechanical-stretch-induced pelvic ligament fibroblasts was implanted in PFD rats. Three weeks later, the HAAM was removed, and Masson staining showed a large amount of matrix
A

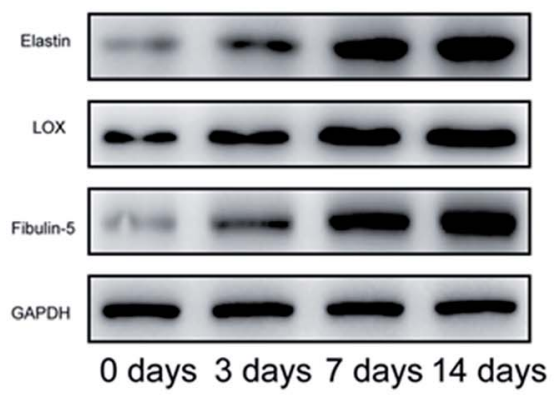

B
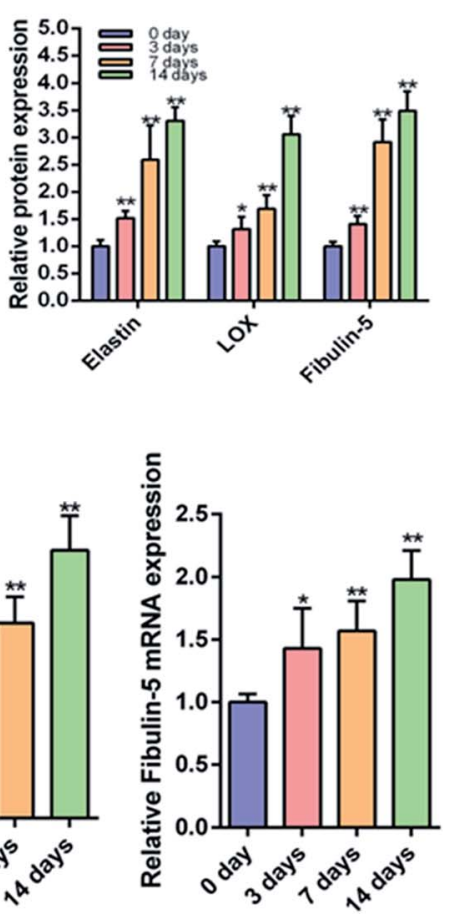

Fig. 4 HAAM promoted the differentiation of BMSCs into pelvic ligament fibroblasts. (A) After HAAM was inoculated with BMSCs and cultured with them for $0,3,7$, or 14 days, western blotting was performed to analyze the expression of elastin, LOX, and fibulin-5 in BMSCs. (B) After HAAM was inoculated with BMSCs and cultured with them for 0, 3, 7, or 14 days, gray-scale analysis was performed to analyze the western blotting results (the expression of elastin, LOX, and fibulin-5 in BMSCs). (C) After BMSCs were inoculated onto and cultured on HAAM for 0, 3, 7, or 14 days, real-time PCR was performed to detect the expression of elastin, LOX, and fibulin-5 in BMSCs. $* P<0.05, * * P<0.01$. 
A

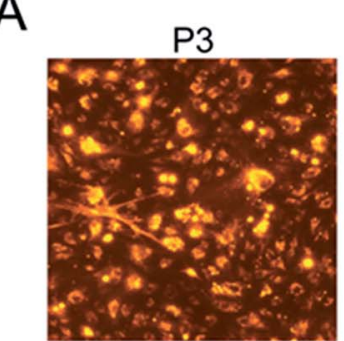

P5

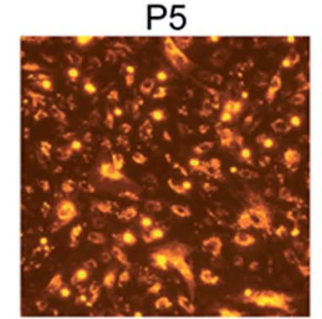

C
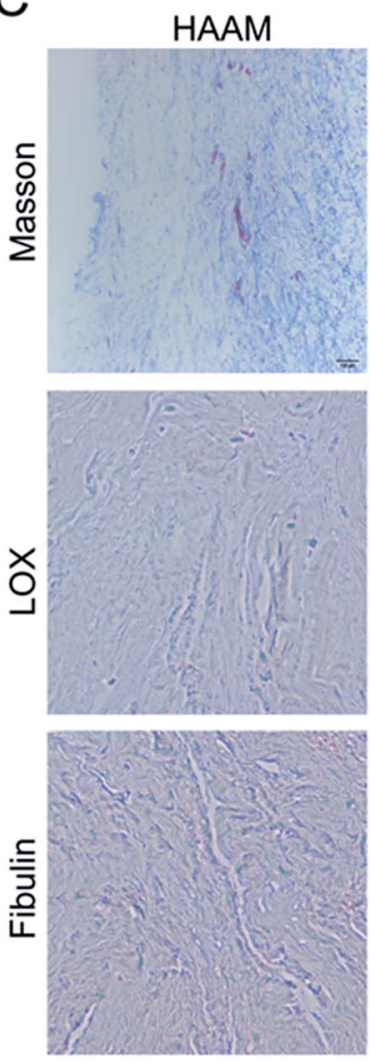

$\mathrm{P} 4$

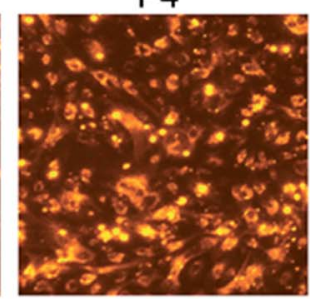

P6

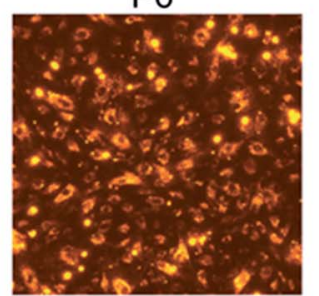

HAAM+BMSC
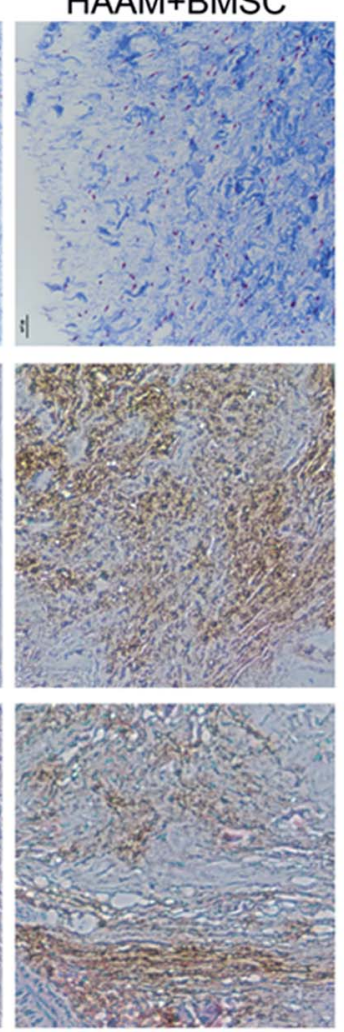

B

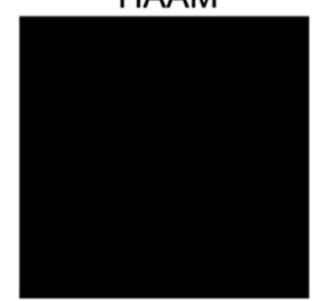

HAAM+ CM-Dil-BMSC

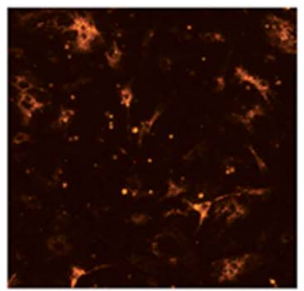

HAAM+ co-culture BMSC
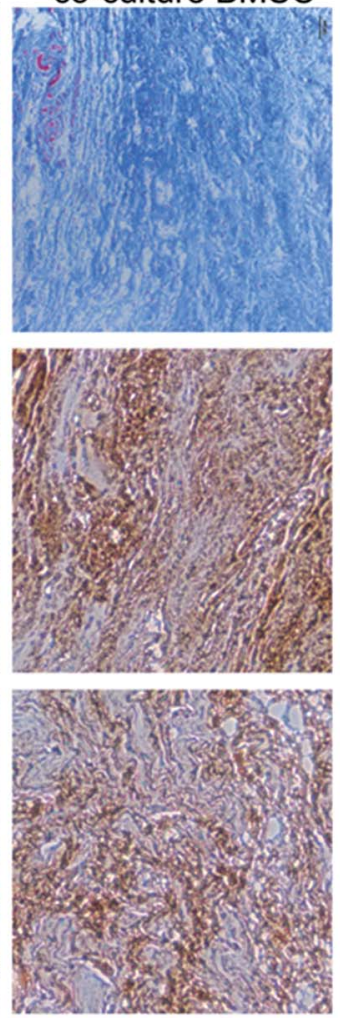

Fig. 5 HAAM loaded with BMSCs cultured with mechanical stretch-stimulated pelvic ligament fibroblasts promoted the production of ligament fibrous tissue in rats. (A) CM-Dil was used to label P3 BMSCs; P3, P4, P5, and P6 BMSCs were observed under a fluorescence microscope. (B) CMDil-labeled BMSCs were seeded on HAAM and then implanted into PFD rats. Three weeks later, a fluorescence microscope was used to observe the survival of BMSCs on HAAM. (C) BMSC-loaded HAAM was then implanted in PFD rats. Three weeks later, HAAM was removed for Masson staining and immunohistochemistry to analyze the expression of LOX and fibulin-5.

collagen in BMSCs at a significantly higher level than that in the control group (implantation of unloaded HAAM) and the other experimental group (implantation of HAAM loaded with undifferentiated BMSCs) (Fig. 5C). HAAM has a good biocompatibility for BMSCs, and could promote the proliferation of BMSCs. The implantation of HAAM loading with BMSCs is beneficial to the growth of BMSCs. The proliferation of BMSCs provides a cell basis for the production of collagen. On the other hand HAAM itself could promote the expression of LOX and fibulin-5 in BMSCs, thus promoting the synthesis of collagen. Immunohistochemical staining showed high expression of LOX and fibulin-5 in the experimental group with implantation of HAAM loaded with BMSCs cultured with mechanical-stretchinduced pelvic ligament fibroblasts. These results suggested 
A

that HAAM loaded with BMSCs cultured with mechanicalstretch-induced pelvic ligament fibroblasts promoted the production of ligament fibrous tissue in rats.

HAAM loaded with BMSCs cultured with mechanical stretchstimulated pelvic ligament fibroblasts improved the function of PFD rats

HAAM loaded with BMSCs cultured with mechanical stretchstimulated pelvic ligament fibroblasts was implanted in PFD rats. Three weeks later, maximum bladder capacity and leak point pressure were significantly increased, and the positive rate of the sneeze test was decreased, which suggested that HAAM loaded with BMSCs cultured with mechanical stretchstimulated pelvic ligament fibroblasts improved the function of PFD rats. Some researchers used BMSCs as seed cells, which were autologous BMSCs that proliferated in vitro, that were loaded onto HAAM (scaffold); the HAAM was then implanted to repair cartilage, where a good healing outcome was observed. In this study, we seeded human BMSCs onto HAAM and found that HAAM promoted the differentiation of BMSCs into ligament fibroblasts. Moreover, the implantation of HAMM, which was loaded with BMSCs cultured with mechanical stretch-induced pelvic ligament fibroblasts, into PFD rats effectively increased the maximum bladder capacity and leak point pressure and decreased the positive rate of the sneeze test, thereby improving the function of PFD rats (Fig. 6A-C).

\section{Conclusion}

This study showed that when cultured with mechanical stretchinduced pelvic ligament fibroblasts, rat BMSCs were induced to differentiate into pelvic ligament fibroblasts. Thus, tissue engineering may provide a new treatment for PFD. BMSCs cultured with mechanical stretch-induced pelvic ligament fibroblasts are ideal seed cells, whereas HAAM is an ideal bioscaffold that promotes BMSC proliferation and differentiation into ligament fibroblasts. Moreover, the implantation of HAMM, which was loaded with BMSCs cultured with mechanical stretch-induced pelvic ligament fibroblasts, into PFD rats effectively improved the function of PFD rats.

\section{Acknowledgements}

This work was supported by the National Natural Science Foundation of China (Grant No. 81300469) and the Medical Science Research Project of Henan Province (No. 21303093). We appreciate all the other staff in the Laboratory Animal Center of Zhengzhou University for their assistance in the experiments.

\section{References}

1 B. Chen and J. Yeh, J. Urol., 2011, 186, 1768-1772.

2 K. Lammers, S. L. Lince, M. A. Spath, L. C. van Kempen, J. C. Hendriks, M. E. Vierhout and K. B. Kluivers, International Urogynecology Journal, 2012, 23, 313-319.

3 C. Marrero, A. Aponte, R. Torres, F. Santos and J. Rivera, P. R. Health Sci. J., 2010, 29, 394-396.

4 J. Alvarez, K. Cvach and P. Dwyer, Minerva Ginecol., 2013, 65, 53-67.

5 M. Jin, Y. Wu, J. Wang, W. Ye, L. Wang, P. Yin, W. Liu, C. Pan and X. Hua, Stem Cell Res. Ther., 2016, 7, 167.

6 M. Jin, Y. Chen, Y. Zhou, Y. Mei, W. Liu, C. Pan and X. Hua, Stem Cell Res. Ther., 2016, 7, 51. 
7 A. N. Smith, E. Willis, V. T. Chan, L. A. Muffley, F. F. Isik, N. S. Gibran and A. M. Hocking, Exp. Cell Res., 2010, 316, 48-54.

8 Z. Bing, L. Linlin, Y. Jianguo, R. Shenshen, R. Ruifang and Z. Xi, Mol. Biol. Rep., 2012, 39, 6077-6085.

9 J. C. Francisco, R. Correa Cunha, M. A. Cardoso, R. Baggio Simeoni, B. F. Mogharbel, G. L. Picharski, D. Silva Moreira Dziedzic, L. C. Guarita-Souza and K. A. Carvalho, Transplant. Proc., 2016, 48, 2845-2849.

10 F. Chehelcheraghi, H. Eimani, S. Homayoonsadraie, G. Torkaman, A. Amini, H. Alavi Majd and H. Shemshadi, Iranian Red Crescent Medical Journal, 2016, 18, e25588.

11 D. D. Rahn, J. F. Acevedo and R. A. Word, Am. J. Physiol.: Regul., Integr. Comp. Physiol., 2008, 295, R1351-R1358.

12 M. Budatha, S. Silva, T. I. Montoya, A. Suzuki, S. ShahSimpson, C. K. Wieslander, M. Yanagisawa, R. A. Word and H. Yanagisawa, PLoS One, 2013, 8, e56376.
13 R. Yang, L. Wu, J. Chen, W. Chen, L. Zhang, L. Zhang, R. You, L. Yin, C. H. Li and Y. Q. Guan, ACS Appl. Mater. Interfaces, 2016, DOI: 10.1021/acsami.6b10377.

14 Q. Zhang, Y. Zhao, Y. Xu, Z. Chen, N. Liu, C. Ke, B. Liu and W. Wu, J. Transl. Med., 2016, 14, 223.

15 X. Liu, C. Bao, H. H. Xu, J. Pan, J. Hu, P. Wang and E. Luo, Acta Biomater., 2016, 42, 378-388.

16 N. Watanabe, S. L. Woo, C. Papageorgiou, C. Celechovsky and S. Takai, Microsc. Res. Tech., 2002, 58, 39-44.

17 H. R. Augsburger and D. Henzi, Theriogenology, 2008, 69, 1070-1076.

18 M. Mahmoudi Rad, F. Talebpour Amiri, M. Mirhoseini, M. Ghasemi, M. Mirzaei and N. Mosaffa, Wounds, 2016, 28, 14-19.

19 J. Yuan, W. Li, J. Huang, X. Guo, X. Li, X. Lu, X. Huang and H. Zhang, Stem Cell Res. Ther., 2015, 6, 217.

20 P. F. Liu, L. Guo, D. W. Zhao, Z. J. Zhang, K. Kang, R. P. Zhu and X. L. Yuan, Genet. Mol. Res., 2014, 13, 7992-8001. 